\title{
A technique for capturing broad subtypes and circulating recombinant forms of HIV-1 based on anionic polymer-coated magnetic beads
}

\author{
AKIKAZU SAKUDO ${ }^{1,2}$ and KAZUYOSHI IKUTA ${ }^{1}$ \\ ${ }^{1}$ Department of Virology, Center for Infectious Disease Control, Research Institute for Microbial \\ Diseases, Osaka University, Osaka 565-0871; ${ }^{2}$ Laboratory of Biometabolic Chemistry, School of \\ Health Sciences, Faculty of Medicine, University of the Ryukyus, Okinawa 903-0215, Japan
}

Received February 13, 2012; Accepted April 23, 2012

DOI: $10.3892 /$ ijmm.2012.1009

\begin{abstract}
Magnetic beads coated with an anionic polymer, poly(methyl vinyl ether-maleic anhydrate) [poly(MVE-MA)], were used in a method to capture human immunodeficiency virus type-1 (HIV-1). The beads were incubated with either HIV-1-infected cell culture medium or plasma from HIV-1 infected individuals and separated from the supernatant by applying a magnetic field. After thorough washing, adsorption of HIV-1 by the beads was confirmed by reverse transcription (RT)-polymerase chain reaction (PCR), real-time PCR, enzyme-linked immunosorbent assay and western blotting. The results confirmed the presence of envelope, polymerase, Nef and the viral genome of HIV-1. Furthermore, various subtypes and circulating recombinant forms (CRFs) of HIV-1 including subtype B, C and CRF01_AE and the immature form of subtype B HIV-1 could be captured. Preincubation with neutralizing antibody against HIV-1 envelope gp41 decreased the capture efficiently, suggesting that poly(MVE-MA) binds HIV-1 via gp41. We believe that this capture procedure will be a valuable tool for detecting various types of HIV-1 in both clinical and experimental samples.
\end{abstract}

\section{Introduction}

Rapid and sensitive detection of viruses in blood is critically important in order to reduce the spread of disease as a result of transfusion (1). Human immunodeficiency virus type-1 (HIV-1) is a major virus linked to transfusion-associated transmission of disease. Although serological detection of antibodies against

Correspondence to: Dr Akikazu Sakudo, Department of Virology, Center for Infectious Disease Control, Research Institute for Microbial Diseases, Osaka University, Yamadaoka 3-1, Suita, Osaka 565-0871, Japan

E-mail: sakudo@biken.osaka-u.ac.jp

Key words: virus, magnetic beads, poly(methyl vinyl ether-maleic anhydrate), maleic, anionic, human immunodeficiency virus type-1
HIV-1 can reduce the risk of disease transmission, a better procedure is urgently required (2-8). For example, during the pre-seroconversion window period the quantity of antibody against the virus is low despite the a high load of HIV-1 present in the blood. Infection with immunovariant viruses and immunosilent carriage cause a similar condition. Recent developments in enzyme-linked immunosorbent assay (ELISA), polymerase chain reaction (PCR) and immunochromatography facilitate the detection of HIV-1 in biological samples (9-12). Nonetheless, the sensitivity of these procedures is insufficient to eliminate the risk of viral transmission.

There are two major limiting factors in the development of a protocol to concentrate HIV-1: i) compatibility with current methods of detection and ii) requirement for a straightforward procedure. Several approaches have been used to increase the concentration of viruses in order to enhance the sensitivity of detection (13-15). For example, ultracentrifugation and polyethylene glycol (PEG) mediated precipitation have been used to concentrate a number of different viruses including HIV-1. Ultracentrifugation is a well-known procedure, but is time-consuming and can increase the false-positive rate when combined with PCR $(12,16)$. Although PEG precipitation is simple and easy to perform, the PEG sometimes interferes with the subsequent PCR (17). One alternative approach is to use magnetic beads coated with molecules that efficiently bind viral particles. Indeed, we and other groups have reported that an anionic polymer, poly(methyl vinyl ether-maleic anhydrate) [poly(MVE-MA)] can be used to capture different viruses.

Here, we report that magnetic beads coated with poly(MVE-MA) are useful for the capture of various subtypes and circulating recombinant form (CRF) of HIV-1. The potential of this method and the mechanisms by which the beads bind HIV-1 are being discussed.

\section{Materials and methods}

Reagents. Unless otherwise specified, chemical reagents were obtained from Sigma-Aldrich (St. Louis, MO) or Wako Pure Chemical Industries, Ltd. (Osaka, Japan). The 300-nm-diameter magnetic particles (reducing sedimentation and offering a broad binding surface) with a high ferrite content (allowing 
separation under a magnetic field) were prepared by grafting of poly(MVE-MA) in dimethyl sulfoxide/phosphate buffer $5 / 95$ solution for $3 \mathrm{~h}$ at $37^{\circ} \mathrm{C}$ (Flavigny et al, American Society for Microbiology $104^{\text {th }}$ General Meeting, 166, 2004). The anionic magnetic beads, Viro-adembeads, were obtained from Ademtech (Pessac, France).

Samples. For analysis, we used either cell culture medium of HIV-1 (LAI or L2)-infected MT4 cells (NIH AIDS Research and Reagent Program) or 293T cells (American Type Culture Collection CRL-11268) transfected with HIV-1 molecular clones (pNL4-3, pBal, pIndie-C1, pL2 and 95TNIH022). In addition, plasma from 4 HIV-1-infected individuals was also used. The plasma from HIV-1-infected individuals at a very early stage of infection was purchased from Alpha Therapeutic Corporation (Calexico, CA). The plasma samples were tested for the following: human hepatitis B virus (HBV) surface antigen (-), anti-HIV-1/HIV-2 (-), HIV-1 by PCR (+), anti-human hepatitis virus (HCV) (-), HCV by PCR (-), HBV by PCR (-), human hepatitis A virus by PCR (-) and parvovirus by PCR (-). These results were reported in the Final Viral Marker Report (Repeat Donors) of Alpha Therapeutic Corporation Consolidated Test Results from Memphis Lab and PCR pooling Lab (Finalized date: 14-Apr-2003).

HIV-1 capture. Viral capture was performed according to the manufacturer's instructions (Ademtech). Briefly, after 2 washes with binding buffer, anionic magnetic beads $(50 \mu 1)$ were further washed twice with phosphate-buffered saline (PBS). Then, $50 \mu 1$ of cell culture medium or plasma diluted with $450 \mu 1$ of PBS was added to the washed beads and incubated for $20 \mathrm{~min}$ at room temperature. A magnetic field was then applied to the tubes containing the magnetic beads. The supernatant was discarded and the beads were thoroughly washed 3 times with PBS. The washed beads were resuspended with PBS and subjected to viral RNA extraction, western blotting or ELISA. After separation, 4 fractions were obtained as follows: i) bead fraction (BD), ii) sample before incubation with the beads (BF), iii) supernatant after incubation (SP) and iv) total sample containing the same quantity $(50 \mu 1)$ of cell culture medium or plasma as BD (TL). The viral capture procedure was typically completed within $30 \mathrm{~min}$.

Capture inhibition by anti-HIV antibody. In order to verify the mechanism of viral capture, HIV-1-infected cell culture media were incubated with anti-HIV-1 Env gp41 antibody, 4E10 (Polymun Scientific Immunbiologische Forschung $\mathrm{GmbH}$ ) or anti- $\alpha$-tubulin, B-5-1-2 (Sigma-Aldrich) for $30 \mathrm{~min}$ at $37^{\circ} \mathrm{C}$ prior to addition of the magnetic beads. The samples were then subjected to bead incubation and magnetic separation as described before.

Western blotting. Each fraction was solubilized in an equal volume of $2 \mathrm{X}$ sodium dodecyl sulfate (SDS) gel-loading buffer [ $90 \mathrm{mM}$ Tris- $\mathrm{HCl}$ (pH 6.8), 10\% mercaptoethanol, 2\% SDS, $0.02 \%$ bromophenol blue and $20 \%$ glycerol], boiled for $5 \mathrm{~min}$ and separated on an SDS-12\% polyacrylamide gel electrophoresis (PAGE) before being electroblotted onto a polyvinylidene difluoride (PVDF) membrane (Hybond-P; Amersham Pharmacia Biotech, Piscataway, NJ) for 60 min at

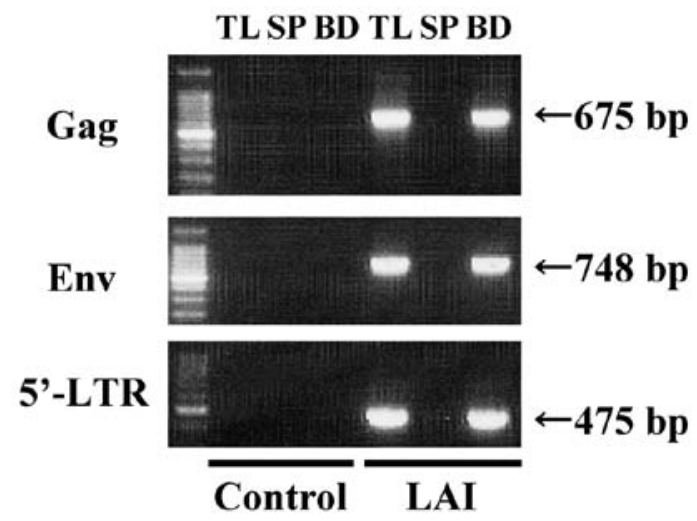

Figure 1. Detection of the RNA genome of HIV-1 adsorbed onto anionic magnetic beads. Culture medium of HIV-1 (LAI)-infected and uninfected (control)-MT4 cells were mixed with anionic magnetic beads. After incubation, the following fractions were obtained: bead fraction (BD), supernatant after incubation (SP) and sample containing the same quantity of culture medium as BD (TL). Viral genomic RNA was extracted from the fractions using a QIAamp viral RNA mini kit and subjected to reverse transcription (RT)-reaction. The resultant cDNAs of HIV-1 Gag protein gene (675 bp), Env protein gene ( $748 \mathrm{bp}$ ) and 5'-LTR (475 bp) were amplified by PCR as described in the Materials and methods. The left lane is the size marker (100 bp ladder).

$15 \mathrm{~V}$. Blots were treated with $5 \%$ skimmed milk for $1 \mathrm{~h}$ at room temperature and then incubated with anti-HIV-1 p24 antibody (03-HIV-18; Biomarket, Ltd.), Nef antibody (clone 2A3, 03-HIV-3; Biomarket, Ltd.), envelope (Env) antibody (SF2 gp120\#387, NIH AIDS Research and Reference Program) and acquired immunodeficiency syndrome (AIDS) patient serum in PBS containing 0.1\% Tween-20 (PBS-T) and 0.5\% skimmed milk for $1 \mathrm{~h}$ at room temperature. After 3 washes with PBS-T, the membrane was incubated in horseradish peroxidase (HRP)-conjugated anti-mouse IgG or anti-human IgG (Jackson ImmunoResearch Laboratories, Inc., West Grove, PA) in PBS-T and $0.5 \%$ skimmed milk for $1 \mathrm{~h}$ at room temperature. After 3 washes with PBS-T, the probed proteins were detected using an enhanced chemiluminescence detection kit (Amersham Pharmacia Biotech).

ELISA. ELISA for HIV-1 p24 was performed using HIV-1 p24 ELISA kit (BioAcademia, Osaka, Japan). Absorbance at $450 \mathrm{~nm}$ was measured to quantify the level of HIV-1 by microplate reader (Labsystems Multiskan MS; Dainippon Sumitomo Pharma Co., Ltd., Osaka, Japan).

Reverse transcription (RT)-PCR. Viral RNA from beads or an aliquot of each sample was extracted with the QIAamp viral RNA mini kit (Qiagen, Hilden, Germany) according to the manufacturer's instructions. RNA was extracted from the magnetic beads by adding lysis buffer prior to removing the beads. RNA was then eluted in $60 \mu \mathrm{l}$ of nuclease-free water. For the RT-reaction, random primers were added and after incubation at $25^{\circ} \mathrm{C}$ for $10 \mathrm{~min}$, the RNA was reverse-transcribed at $65^{\circ} \mathrm{C}$ for $50 \mathrm{~min}$ followed by denaturation of the enzyme at $85^{\circ} \mathrm{C}$ for $5 \mathrm{~min}$. The diluted cDNA was amplified in a reaction mixture containing primers, Ex Taq (Takara Bio, Inc., Otsu, Japan) and Ex Taq buffer under conditions of 30 cycles of $94^{\circ} \mathrm{C}$ for $1 \mathrm{~min}, 60^{\circ} \mathrm{C}$ for $1 \mathrm{~min}$ and $72^{\circ} \mathrm{C}$ for $1 \mathrm{~min}$. PCR was carried out using the following primers for the HIV-1 Gag 

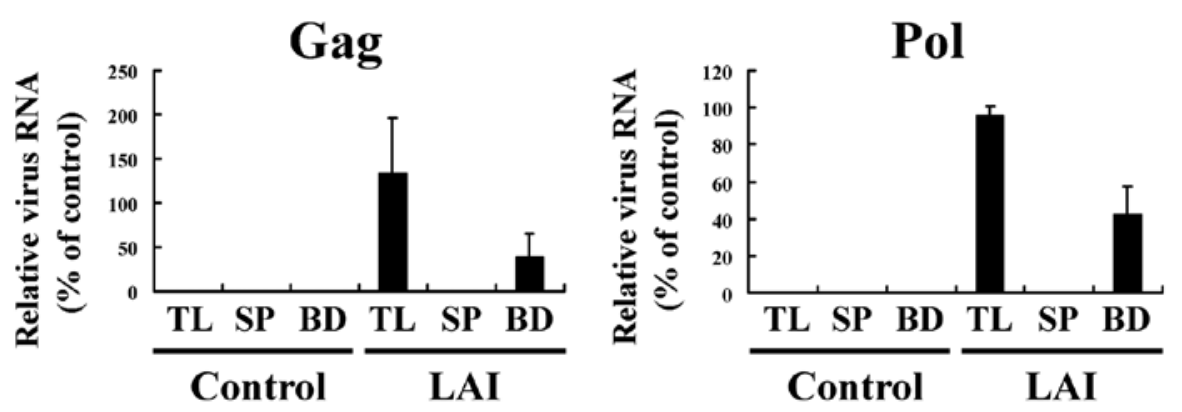

Figure 2. Quantitative analysis of viral protein (Gag and Pol) genes of HIV-1 adsorbed onto anionic magnetic beads. (A) Anionic magnetic beads were used to capture HIV-1 from $500 \mu 1$ of culture medium of cells infected with HIV-1 (LAI) and then analyzed by real-time PCR. Uninfected cells were used as a negative control. Samples were divided into 3 categories: i) bead fraction (BD), ii) supernatant after the incubation (SP) and iii) sample containing the same quantity of culture medium as BD (TL). RNA was purified using a QIAamp viral RNA mini kit, reverse-transcribed and then analyzed by real-time PCR with HIV-1 Gag protein gene $(\mathrm{Gag})$ and $\mathrm{Pol}$ protein gene $(\mathrm{Pol})$.

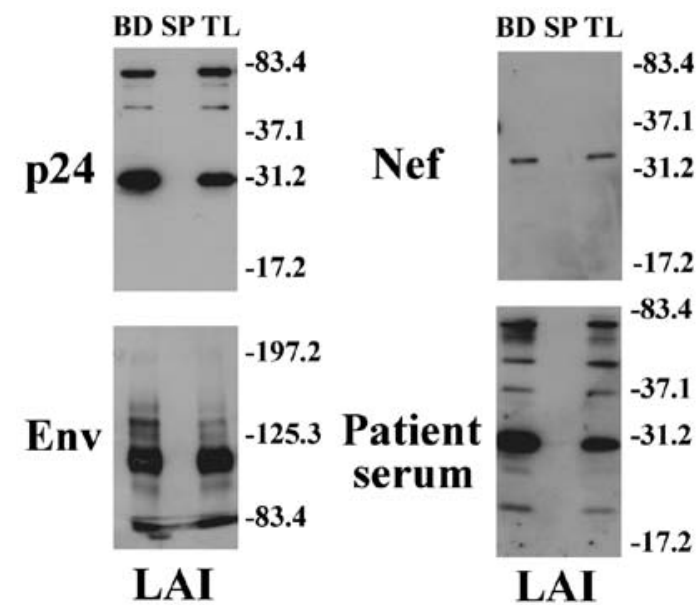

Figure 3. Detection of viral proteins of HIV-1 adsorbed onto anionic magnetic beads. Culture medium of HIV-1 (LAI)-infected MT4 cells was diluted with PBS and subjected to incubation with anionic magnetic beads. Western blotting was performed with anti-HIV-1 p24, Nef and Env antibody and AIDS patient serum. Samples were divided into 3 categories: i) bead fraction (BD), ii) supernatant after the incubation (SP) and iii) total sample containing the same quantity of nasal aspirate as $\mathrm{BD}(\mathrm{TL})$. The samples were solubilized with SDS loading buffer and then subjected to western blotting. Molecular weight marker $(\mathrm{KDa})$ is shown in the right lane.

gene: HIV Gag RTPCR AE B common F, 5'-ggggaagtgacatagcagga-3' and R, 5'-ctgttggctctggtctgctc-3'; for the HIV-1 Env gene: HIV Env RTPCR AE B common F: 5'-gacggtacaggccag acaat-3' and R, 5'-tcccagaagttccacaatcc-3'; for the HIV-1 5'long terminal repeat (LTR): HIV 5'LTR RTPCR AE B common F, 5'-ccetgattggcagaactacac-3' and R, 5'-agcactcaaggcaagcttta-3'. The amplified products were purified and cloned in pT7Blue T-vector (Novagen, Madison, WI). DNA sequencing (ABI PRISM3100 Genetic Analyzer; Applied Biosystems, Foster City, CA) with the R-20mer primer and U-19mer primer (Novagen) was used to verify the product sequence.

Real-time RT-PCR. The cDNAs produced in the RT reactions above were also analyzed by real-time PCR (Q-PCR, quantitative PCR). For real-time PCR, a Brilliant SYBR-Green Q-PCR mastermix was used according to the manufacturer's instructions (Stratagene, La Jolla, CA). Briefly, the Q-PCR components included Brilliant Q-PCR mastermix, reverse- transcribed cDNA, and the forward and reverse target gene primers: realHIVgag F, 5'-caagcagggagctagaacga-3' and R, 5'-ttgtctacagccttctgatgtctc-3'; realHIVpol F, 5'-aaattcaaaattttcgggtttattac-3' and R, 5'-aggagctttgctggtccttt-3'. The Q-PCR program used in a Mx3000 ${ }^{\mathrm{TM}}$ Real-time Q-PCR System (Stratagene) was: denaturation (at $95^{\circ} \mathrm{C}$ for $10 \mathrm{~min}$ ) and then 40 cycles of denaturation $\left(95^{\circ} \mathrm{C}\right.$ for $\left.30 \mathrm{sec}\right)$, annealing $\left(58^{\circ} \mathrm{C}\right.$ for $60 \mathrm{sec})$ and extension $\left(72^{\circ} \mathrm{C}\right.$ for $\left.30 \mathrm{sec}\right)$. Each reaction was done in triplicate. The results were analyzed using the $\mathrm{Mx} 3000 \mathrm{P}^{\mathrm{TM}}$ system software. The relative expression ratio of each sample was calculated using a mathematical model based on the amplification efficiency. PCR specificity was verified by dissociation curve analysis of the amplified DNA fragments.

\section{Results}

To investigate whether Viro-Adembeads could be used to capture HIV-1, cell culture medium from HIV-1 (LAI)infected MT4 cells was mixed with the anionic polymer-coated magnetic beads. The mixture was then magnetically separated and bead (BD), supernatant (SP) and total (TL) fractions were prepared. Cell culture medium from mock-infected MT4 cells was also used to prepare control fractions. The fractions were then analyzed by RT-PCR, real-time PCR, western blotting and ELISA to determine the extent of HIV-1 capture by the beads.

Firstly, RT-PCR was performed to detect HIV-1 genomic RNA in order to examine the capacity of the beads to capture HIV-1 (Fig. 1). RT-PCR analysis gave a single band of $675 \mathrm{bp}$ for Gag, 748 bp for Env and 475 bp for 5'-LTR in the bead fraction (BD) and samples containing the same quantity of cell culture medium as BD (TL) using cell culture medium from HIV-1 (LAI)-infected MT-4 cells. No signal was detected in the supernatant after incubation (SP). In contrast, RT-PCR analysis of $\mathrm{BD}, \mathrm{SP}$ and TL using cell culture medium obtained from Mock-infected MT4 cells (control) did not give any signal. The 675-, 748- and 475-bp bands were confirmed to be Gag, Env and 5'-LTR gene of HIV-1, respectively by DNA sequencing (identity to Genebank accession number AF324493 was 96, 98 and $99 \%$, respectively). Therefore, these results confirm that the bead fraction includes the HIV-1 genomic RNA.

The amount of HIV-1 genomic RNA in the BD, SP and TL fractions was measured by real-time RT-PCR, relative to that in a control (a TL sample with the highest value was taken as 


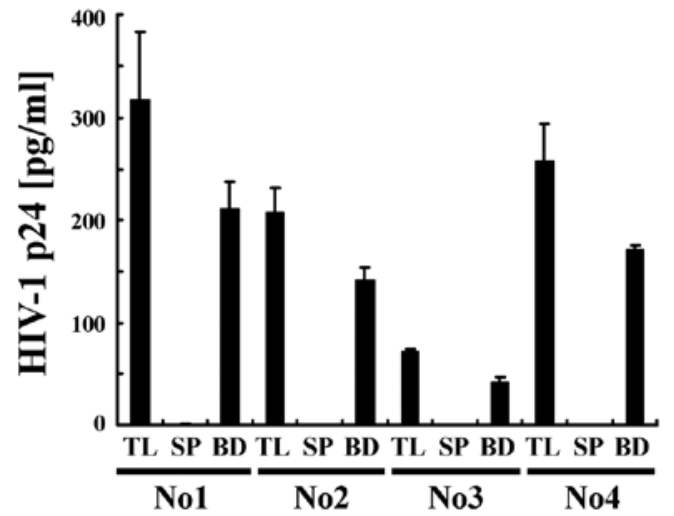

Figure 4. Quantitative analysis of HIV-1 adsorbed onto anionic beads. Plasma from 4 HIV-1-infected individuals (No. 1-4) were diluted with PBS and subjected to incubation with anionic beads. HIV-1 p24 was adsorbed onto the beads using HIV-1 p24 ELISA kit. Samples were divided into 3 categories: i) bead fraction (BD), ii) supernatant after incubation (SP) and iii) sample containing the same quantity of culture medium as BD (TL). The concentration of HIV-1 in each fraction was calculated as an index of absorbance at $450 \mathrm{~nm}$ by comparison with HIV-1 p24 standard.

100 [\%]). For HIV-1 (LAI)-infected cell culture medium, the amount of viral RNA in the BD fraction was $\sim 33$ and 50\% that in the TL from real-time PCR of Gag and Pol genes, respectively (Fig. 2). In contrast, cell culture medium from mock-infected MT4 cells (control) showed no amplification of genomic RNA in BD, SP and TL. The specificity of these PCR reactions was confirmed by dissociation curve analysis of the reaction products. These results showed that HIV-1 in cell culture medium could be captured by the magnetic beads, but that a fraction of HIV-1 was lost during the procedure. A significant fraction of HIV-1 in the culture medium of virusinfected cells was lost during the capture procedure using anionic polymer-coated magnetic beads. The loss of HIV-1 may have been due to serum components in the cell culture medium, such as albumin, binding to the magnetic beads and thereby hindering viral capture (18).

Western blotting demonstrated that the total sample fraction (TL) and bead fraction (BD), but not the supernatant fraction (SP), in cell culture medium of HIV-1 (LAI)-infected MT4 cells had a major band of 30,34 and $110 \mathrm{kDa}$ for p24, Nef and Env proteins, respectively (Fig. 3). These bands correspond to the respective deduced mass of HIV-1 p24, Nef and Env protein based on their amino acid sequences. Thus, HIV-1 was detected at similar levels in the total sample fraction (TL) and in the bead fraction (BD), but not at all in the supernatant fraction (SP). In addition, the corresponding bands were detected using serum from an AIDS patient. These results support the idea that HIV-1 is efficiently captured by anionic magnetic beads.

Next, we examined the efficiency with which HIV-1 was captured from plasma by conducting a quantitative analysis using ELISA (Fig. 4). HIV-1 in plasma from 4 HIV-1-infected individuals (No. 1-4) was recovered using anionic magnetic beads (BD) at a level of $65-80 \%$ that from samples containing the same quantity of plasma as BD (TL). In contrast, HIV-1 was below the detection limit in the supernatant after incubation (SP). These findings suggest that most of the HIV-1 was efficiently captured from plasma by the magnetic beads.

To further examine whether this magnetic capture method can be applied to broad subtypes of HIV-1, cell culture medium of 293T cells transfected with various types of HIV-1 molecular clones, such as pNL4-3 (subtype B), pBal (subtype B), pIndie-C1 (subtype C) and 95TNIH022 (CRF01_AE), were subjected to magnetic capture (Fig. 5). ELISA showed that HIV-1 from cell culture media of 293T cells transfected with HIV-1 pNL4-3, pBal or pIndie- $\mathrm{C} 1$ could be captured by magnetic beads at a similar level of capture efficiently (60-80\%). However, cell culture medium of 293T cells transfected with 95TNIH022, showed a lower efficiency of HIV-1 capture compared to the molecular clones of subtype B and C. This may be due to the overall lower concentration (about 100-fold lower) of HIV-1 in cell culture of $293 \mathrm{~T}$ cells transfected with 95TNIH022 compared to the other molecular clones.

Next, we used gp120-containing, protease-deficient clone (L2), which is derived from LAI and generate immature and defective doughnut-shaped particles (19) (Fig. 6 and 7). ELISA (Fig. 6) and western blotting (Fig. 7) showed that HIV-1 produced by transfection of pL2 into $293 \mathrm{~T}$ cells could be efficiently captured by magnetic beads. Although L2 expresses an immature form of polymerase and decreased levels of Env compared to wild type LAI, the L2 polymerase and Env were efficiently captured by anionic beads. Finally, we investigated the mechanisms by which poly(MVE-MA) binds HIV-1. HIV-1 LAI in cell culture medium was preincubated with antiHIV-1 Env gp41 neutralizing antibody 4E10 before incubation
A

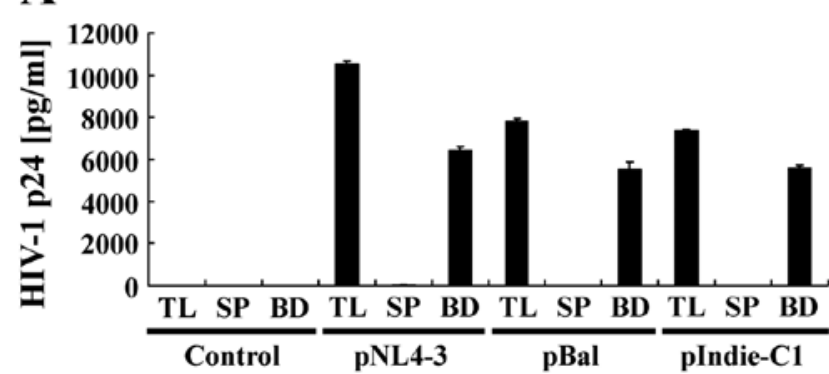

B

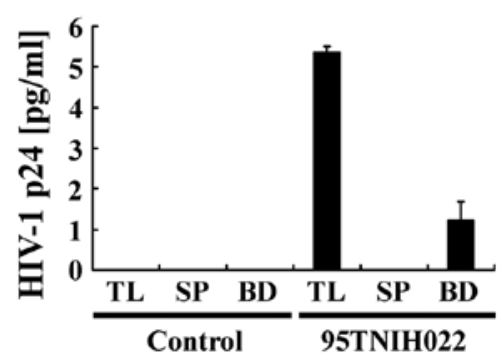

Figure 5. Adsorption of HIV-1 with subtype B, C and circulating recombinant form CRF_A E onto anionic magnetic beads. (A) HIV-1 in cell culture medium of $293 \mathrm{~T}$ cells transfected with HIV-1 molecular clone of pNL4-3, pBal and pIndie-C1 or (B) MT-4 cells infected with HIV-1 95 TNIH022 before and after adsorption onto anionic magnetic beads were quantitatively analyzed by ELISA using HIV-1 p24 ELISA kit. Samples were divided into 3 categories: i) bead fraction (BD), ii) supernatant after the incubation (SP) and iii) sample containing the same quantity of culture medium as BD (TL). 


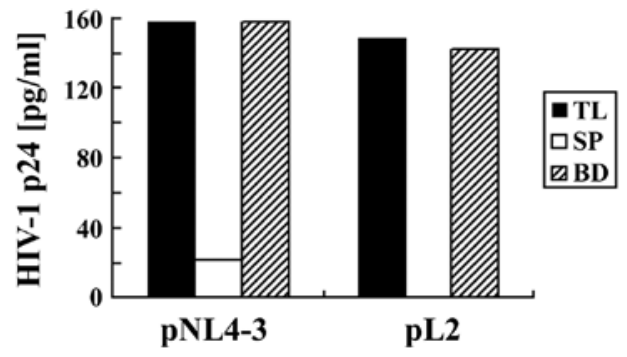

Figure 6. Quantitative analysis of adsorption of HIV-1 produced by transfection of HIV-1 molecular clone onto anionic beads. HIV-1 in cell culture medium of 293T cells transfected with HIV-1 molecular clone pNL4-3 and pL2 before and after adsorption on anionic magnetic beads was quantitatively analyzed by ELISA using HIV-1 p24 ELISA kit. Samples were divided into 3 categories: i) bead fraction (BD), ii) supernatant after the incubation (SP) and iii) sample containing the same quantity of nasal aspirate as BD (TL). The concentration of HIV-1 in each fraction was calculated as an index of absorbance at $450 \mathrm{~nm}$ by comparison with HIV-1 p24 standard.

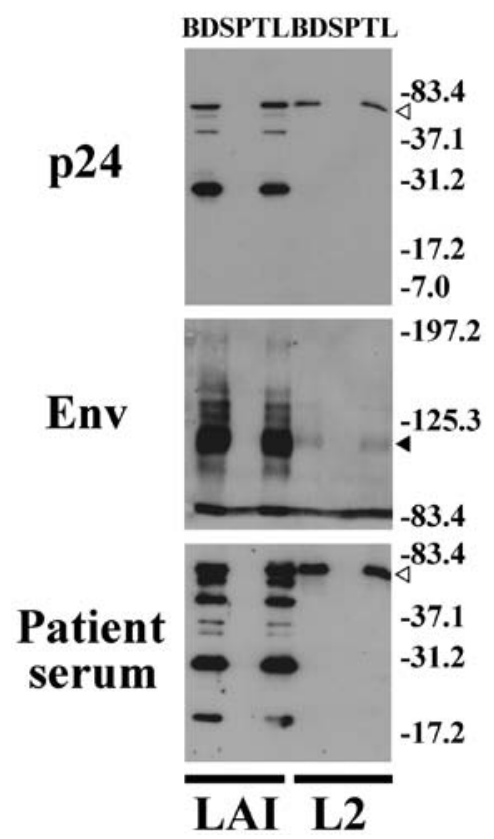

Figure 7. Detection of viral proteins of HIV-1 and the gp120-containing, protease-deficient clone (L-2) adsorbed onto anionic magnetic beads. Culture medium of HIV-1 (LAI or L2)-infected MT4 cells was diluted with PBS and subjected to incubation with anionic magnetic beads. Western blotting was performed with anti-HIV-1 p24 and Env antibody and AIDS patient serum. Samples were divided into 3 categories: i) bead fraction (BD) (ii) supernatant after the incubation (SP) and iii) sample containing the same quantity of nasal aspirate as $\mathrm{BD}(\mathrm{TL})$. L2 shows immature polymerase (white arrow heads) and low expression of Env protein (black arrow head).

with the magnetic beads (Fig. 8). Our results revealed that antiHIV-1 Env antibody 4E10 inhibited the binding of HIV-1 to the magnetic beads, whereas the anti-tubulin antibody, which was used as a negative control, showed no such inhibition.

\section{Discussion}

The magnetic bead-mediated capture method for HIV-1 is a simple and quick procedure that significantly reduces problems associated with loss of signal and possible cross-contamination commonly observed in multi-step protocols. Moreover, the

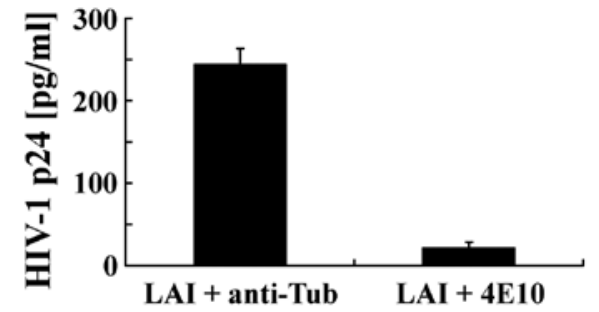

Figure 8. Inhibition of adsorption of HIV-1 onto anionic magnetic beads by preincubation with anti-HIV-1 Env gp41 neutralizing antibody. Culture medium of HIV-1 (LAI)-infected MT-4 cells was incubated with anti-HIV-1 Env gp41 antibody 4E10 or anti- $\alpha$-tubulin antibody B5-1-2 for $30 \mathrm{~min}$ at $37^{\circ} \mathrm{C}$. After the incubation, anionic magnetic beads were added and further incubated for $20 \mathrm{~min}$. The beads were then collected by application of a magnetic field and subjected to HIV-1 p24 ELISA.

magnetic bead-mediated capture method can be applied to a range of sample types, such as cell culture and plasma as well as other body fluids, and is compatible with conventional detection methods, such as PCR, ELISA and western blotting. Indeed, previously described methods for concentrating viruses are often incompatible with conventional detection procedures $(12,16,17)$. For example, the ultracentrifugation procedure requires expensive specialist equipment and is relatively timeconsuming compared to the magnetic bead-mediated capture method. PEG precipitation is sometimes incompatible with PCR due to PEG mediated inhibition of the DNA polymerase. Therefore, capture by magnetic beads is a promising approach compared to previous virus concentrating methods. There are several methods for concentrating a virus using magnetic beads coated with an antibody for a specific virus $(16,20,21)$ and polymers such as polyethyleneimine (PEI) for simian virus 40 (SV40) (22), herpes simplex virus type 1 (HSV-1) (22), Sindbis virus (22), vesicular stomatitis virus (VSV) (22), amphotropic murine leukemia virus (23), poliovirus (24), hepatitis A virus (HAV) (24), hepatitis B virus (HBV) (24), hepatitis C virus (HCV) (24), and cytomegalovirus (CMV) (25) or sulfonated (SO) magnetic beads in the presence of divalent cations for cytomegalovirus (25), Sindbis virus (25), poliovirus (25) and porcine parvovirus (25). Moreover, poly(MVE-MA)-coated magnetic beads can be used for efficient capture of avian and human influenza virus $(18,26)$, respiratory syncytial virus (27), Borna disease virus (28), dengue virus (29), HIV-1 subtype B (30), CMV (30), Rota virus (30), herpesvirus (31) and vaccinia virus (31).

The present study clearly shows that poly(MVE-MA)coated magnetic beads can be used to capture HIV-1 with various subtypes and CRF. Although the mechanism by which the magnetic beads bind to HIV-1 remains unclear, Env may be involved in this process because preincubation of the beads with neutralizing antibody against HIV-1 Env prevented capture of HIV-1. Electrostatic, hydrophilic, hydrophobic and steric organization of poly(MVE-MA) may also contribute to the binding (18). Because poly(MVE-MA) is negatively charged, modification of the spatial organization of the polymers may change binding efficiency and capacity of HIV-1 (32). Therefore, charge density and steric spatial organization may provide some information on the binding mechanism. However, we also show that the immature form of HIV-1 L2 with low production of Env can also be captured, suggesting that the beads can bind to HIV-1 by a mechanism independent 
of the Env expression level. The 20-35\% loss of HIV-1 during bead capture from HIV-1-infected plasma may be due to nonspecific binding of albumin and immunoglobulin as previously shown (18). However, modification of charge density and surface organization of polymer may reduce the non-specific binding.

In conclusion, we demonstrated that magnetic beads coated with an anionic polymer are useful for the capture and concentration of HIV-1. In the captured HIV-1, the presence of a viral genome, Env, polymerase and Nef were confirmed by RT-PCR, real-time PCR, ELISA and western blotting. Therefore, this method can be used in combination with conventional means of detection. The applicability of this method to different types of viruses is currently being studied.

\section{Acknowledgements}

The authors thank Mr. Takanori Kobayashi for technical assistance as well as Ms. Etsuko Kita and Yurie Shimada for their administrative support. This work was supported by Grant-in-Aid for Promotion of Basic Research Activities for Innovative Biosciences from Bio-oriented Technology Research Advancement Institution (BRAIN) and for Scientific Research on Innovative Areas from Japan Society for the Promotion of Science.

\section{References}

1. Schreiber GB, Busch MP, Kleinman SH and Korelitz JJ: The risk of transfusion-transmitted viral infections. The Retrovirus Epidemiology Donor Study. N Engl J Med 334: 1685-1690, 1996.

2. Candotti D, Adu-Sarkodie Y, Davies F, et al: AIDS in an HIV-seronegative Ghanaian woman with intersubtype A/G recombinant HIV-1 infection. J Med Virol 62: 1-8, 2000.

3. Germain M, Gelinas S and Delage G: Estimates of risk of window-period transmission of blood-borne viral diseases in Quebec. CMAJ 170: 1077-1078, 2004.

4. Loussert-Ajaka I, Ly TD, Chaix ML, et al: HIV-1/HIV-2 seronegativity in HIV-1 subtype O infected patients. Lancet 343 : 1393-1394, 1994.

5. Phelps R, Robbins K, Liberti T, et al: Window-period human immunodeficiency virus transmission to two recipients by an adolescent blood donor. Transfusion 44: 929-933, 2004.

6. Soldan K, Barbara JA, Ramsay ME and Hall AJ: Estimation of the risk of hepatitis $B$ virus, hepatitis $C$ virus and human immunodeficiency virus infectious donations entering the blood supply in England, 1993-2001. Vox Sang 84: 274-286, 2003.

7. Granade TC, Workman S, Wells SK, et al: Rapid detection and differentiation of antibodies to HIV-1 and HIV-2 using multivalent antigens and magnetic immunochromatography testing. Clin Vaccine Immunol 17: 1034-1039, 2010.

8. Schramm W, Angulo GB, Torres PC and Burgess-Cassler A: A simple saliva-based test for detecting antibodies to human immunodeficiency virus. Clin Diagn Lab Immunol 6: 577-580, 1999.

9. Jackson BR, Busch MP, Stramer SL and AuBuchon JP: The costeffectiveness of NAT for HIV, HCV, and HBV in whole-blood donations. Transfusion 43: 721-729, 2003.

10. Klein SA, Karsten S, Ruster B, et al: Comparison of TaqMan real-time PCR and p24 ELISA for quantification of in vitro HIV-1 replication. J Virol Methods 107: 169-175, 2003.

11. Cunningham P, Marriott D, Harris C, et al: False negative HIV-1 proviral DNA polymerase chain reaction in a patient with primary infection acquired in Thailand. J Clin Virol 26: 163-169, 2003.

12. Roth WK, Weber M and Seifried E: Feasibility and efficacy of routine PCR screening of blood donations for hepatitis $\mathrm{C}$ virus, hepatitis B virus, and HIV-1 in a blood-bank setting. Lancet 353: 359-363, 1999.
13. Kittigul L, Khamoun P, Sujirarat D, Utrarachkij F, Chitpirom K, Chaichantanakit $\mathrm{N}$ and Vathanophas K: An improved method for concentrating rotavirus from water samples. Mem Inst Oswaldo Cruz 96: 815-821, 2001.

14. Sanyal D, Kudesia G and Corbitt G: Comparison of ultracentrifugation and polyethylene glycol precipitation for concentration of hepatitis B virus (HBV) DNA for molecular hybridisation tests and the relationship of HBV-DNA to $\mathrm{HBe}$ antigen and anti- $\mathrm{HBe}$ status. J Med Microbiol 35: 291-293, 1991.

15. Trepanier P, Payment $P$ and Trudel M: Concentration of human respiratory syncytial virus using ammonium sulfate, polyethylene glycol or hollow fiber ultrafiltration. J Virol Methods 3: 201-211, 1981.

16. Kobayashi S, Natori K, Takeda $\mathrm{N}$ and Sakae K: Immunomagnetic capture rt-PCR for detection of norovirus from foods implicated in a foodborne outbreak. Microbiol Immunol 48: 201-204, 2004.

17. Novotný J, Svobodová J, Ransnäs LA and Kubistová K: A method for the preparation of purified antigens of coxsackievirus B3 from a large volume of cell culture supernatant. Acta Virol 36: 483-487, 1992.

18. Sakudo A, Baba K, Tsukamoto M, et al: Anionic polymer, poly(methyl vinyl ether-maleic anhydride)-coated beads-based capture of human influenza A and B virus. Bioorg Med Chem 17: 752-757, 2009.

19. Ohki K, Kishi Y, Nishino Y, et al: Noninfectious doughnutshaped human immunodeficiency virus type 1 can induce syncytia mediated by fusion of the particles with CD4-positive cells. J Acquir Immune Defic Syndr 4: 1233-1240, 1991.

20. Clavet CR, Margolin AB and Regan PM: Herpes simplex virus type-2 specific glycoprotein G-2 immunomagnetically captured from HEp-2 infected tissue culture extracts. J Virol Methods 119: 121-128, 2004.

21. Jothikumar N, Cliver DO and Mariam TW: Immunomagnetic capture PCR for rapid concentration and detection of hepatitis A virus from environmental samples. Appl Environ Microbiol 64: 504-508, 1998.

22. Satoh K, Iwata A, Murata M, et al: Virus concentration using polyethyleneimine-conjugated magnetic beads for improving the sensitivity of nucleic acid amplification tests. J Virol Methods 114: 11-19, 2003.

23. Uchida E, Sato K, Iwata A, et al: An improved method for detection of replication-competent retrovirus in retrovirus vector products. Biologicals 32: 139-146, 2004.

24. Uchida E, Kogi M, Oshizawa T, et al: Optimization of the virus concentration method using polyethyleneimine-conjugated magnetic beads and its application to the detection of human hepatitis A, B and C viruses. J Virol Methods 143: 95-103, 2007.

25. Iwata A, Satoh K, Murata M, et al: Virus concentration using sulfonated magnetic beads to improve sensitivity in nucleic acid amplification tests. Biol Pharm Bull 26: 1065-1069, 2003.

26. Sakudo A and Ikuta K: Efficient capture of infectious H5 avian influenza virus utilizing magnetic beads coated with anionic polymer. Biochem Biophys Res Commun 377: 85-88, 2008.

27. Sakudo A, Baba K, Tsukamoto M and Ikuta K: Use of anionic polymer, poly(methyl vinyl ether-maleic anhydride)-coated beads for capture of respiratory syncytial virus. Bioorg Med Chem Lett 19: 4488-4491, 2009.

28. Sakudo A, Tanaka Y and Ikuta K: Capture of infectious borna disease virus using anionic polymer-coated magnetic beads. Neurosci Lett 494: 237-239, 2011.

29. Sakudo A, Masrinoul P, Tanaka Y and Ikuta K: Capture of dengue virus type 3 using anionic polymer-coated magnetic beads. Int J Mol Med 28: 625-628, 2011.

30. Ademtech: Viro-Adembeads: virus capture and culture. http:// www.ademtech.com/images/viro-adembeads.pdf. Accessed January 4, 2012.

31. Hatano B, Kojima A, Sata T and Katano H: Virus detection using Viro-Adembeads, a rapid capture system for viruses, and plaque assay in intentionally virus-contaminated beverages. Jpn J Infect Dis 63: 52-54, 2010.

32. Sakudo A and Onodera T: Virus capture using anionic polymercoated magnetic beads (Review). Int J Mol Med 30: 3-7, 2012. 